\title{
Shear banding and flow-concentration coupling in colloidal glasses
}

\author{
R. Besseling ${ }^{1}$, L. Isa ${ }^{1}$, P. Ballesta ${ }^{2}$, G. Petekidis ${ }^{2}$, M. E. Cates ${ }^{1}$ and W. C. K. Poon ${ }^{1}$ \\ ${ }^{1}$ SUPA, School of Physics \& Astronomy, The University of Edinburgh, \\ Kings Buildings, Mayfield Road, Edinburgh EH9 3JZ, United Kingdom. \\ 2 IESL-FORTH and Department of Materials Science and Technology, \\ University of Crete, Heraklion 71110, Crete, Greece.
}

\begin{abstract}
We report experiments on hard sphere colloidal glasses that show a type of shear banding hitherto unobserved in soft glasses. We present a scenario that relates this to an instability due to shearconcentration coupling, a mechanism previously thought unimportant in these materials. Below a characteristic shear rate $\dot{\gamma}_{c}$ we observe increasingly non-linear and localized velocity profiles. We attribute this to very slight concentration gradients in the unstable flow regime. A simple model accounts for both the observed increase of $\dot{\gamma}_{c}$ with concentration, and the fluctuations in the flow.
\end{abstract}

PACS numbers: 83.60.-a, 83.50.-v, 81.40.Lm, 83.80.Hj

Shear banding is widespread in the flow of disordered materials, including complex fluids [1]; pastes, gels and emulsions [2]; granular matter [3], soils and rocks [4] and metallic glasses [5]. Understanding this phenomenon is thus crucial in various fields of science and engineering.

Constitutive models of shear banding exist, e.g. in wormlike micellar fluids [1], where a flow curve of stress versus strain rate $\sigma(\dot{\gamma})$ with slope $\eta_{d}<0$ causes instability and separation into two bands with distinct flow rates $\left(\dot{\gamma}_{1}, \dot{\gamma}_{2}\right)>0$ [1]; these can also have distinct concentrations [6]. Many systems with a yield stress $\left(\sigma_{y}>0\right)$ also show coexistence of distinct bands [7-10], with one band now being solid $\left(\dot{\gamma}_{1}=0\right)$. In some cases this stems from a similar mechanical instability $\left(\eta_{d}<0\right.$ for $\left.\dot{\gamma}<\dot{\gamma}_{2}\right)$ [9-12] due to positive feedback between flow and structural breakup. However the banding seen in other experiments [13, 14, 16, 17] and simulations [18, 19], cannot be explained in those terms, particularly for purely repulsive interactions [20]. In some of these systems, banding may be attributed to cooperativity between local plastic events, characterized by a cooperativity length $\xi$. However, theory 21] and simulations 222] show that $\xi$ grows when the flow slows down, which contrasts with the rateindependence seen in experiments [16].

In this Letter we show by experiment and theory that concentrated hard-sphere (HS) colloids, one of the simplest yield-stress fluids and a model for soft glasses generally, can exhibit a type of shear banding that does not fit into any of the above categories. Instead, we propose a scenario where banding is caused by shear-concentration coupling (SCC). Though well known as a generic mechanism for flow instability [23], this has not previously been explored as a shear banding mechanism in glasses. This is perhaps because the concentration changes involved can be extremely small, as we show below; hence they are not directly detectable in experiments. Crucially, the effects on flow of very small concentration gradients are vastly amplified by the presence of a yield stress.

It is well known that for nonuniform stress, particle migration [24] is driven by gradients in $\dot{\gamma}$ and the nonequilibrium particle pressure, $\Pi(\dot{\gamma})$ [25, 26]. The resulting concentration inhomogeneity causes departures from flow profiles for the homogeneous system. But this can not explain such departures under uniform stress, which requires intrinsic instability [23] (see also [24]). Recent theories 27] show that Newtonian and linearly viscoelastic materials can also exhibit such instability via SCC. However, this arises at $45^{\circ}$ to the flow direction, and is unrelated to the results in 23] on SCC-induced instability in nonlinear fluids. In applying the latter to glasses, our two key ingredients are (non-linear) dilatancy -the tendency of jammed systems to expand under flow, $(\partial \Pi / \partial \dot{\gamma}>0)$ and flow nonlinearity. As both are ubiquitous in glassy materials, our results are likely to have wide relevance.

We used sterically stabilised polymethylmethacrylate particles (radii $a=138 \mathrm{~nm}$ and $150 \mathrm{~nm}$, polydispersity $\sim 15 \%$ ) suspended in a decalin-tetralin mixture (viscosity $\left.\eta_{s}=2.3 \mathrm{mPas}\right)$ and seeded with $\sim 0.5 \%$ fluorescent colloids $(a=652 \mathrm{~nm})$ of the same kind. Different volume fractions $\phi$ were prepared by diluting samples centrifuged to a sediment with $\phi=\phi_{m} \simeq 0.67$; we report data in terms of the reduced concentration $\Phi=\phi / \phi_{m}$. The glass transition was found to lie at $\Phi_{g} \simeq 0.86$ [28].

Rheology was measured in an AR2000 rheometer in cone/transparent plate geometry (cone angle $1^{\circ}$, radius $20 \mathrm{~mm}$ ), coupled to a confocal microscope [29, 30] to measure the velocity $v(z)$ across the gap, $0 \leq z \leq z_{g}$, at various $z_{g}$. For $\dot{\gamma} \gtrsim 0.1 \mathrm{~s}^{-1}$ we measured $v(z)$ at each $z$ for a time $\geq 3 / \dot{\gamma}$; for $\dot{\gamma} \lesssim 0.1 \mathrm{~s}^{-1}, v(z)$ was measured from rapid 3D scans [30] over a time $\sim 2 / \dot{\gamma}$. The reported $v(z)$ are constant over the measurement time. To prevent slip [29], walls were coated with a disordered monolayer of the tracers; results for a cone and/or plate roughened to $\sim 10 \mu \mathrm{m}$ were very similar. We took data at fixed $\dot{\gamma}$, stepping down $\dot{\gamma}$ after 300 s preshear at $\dot{\gamma} \simeq 10-30 \mathrm{~s}^{-1}$ or stepping up from $\dot{\gamma}=0$ (waiting for $\geq 2 / \dot{\gamma}$ before acquiring $v(z)$ in either case). The results showed no systematic differences between these protocols; stress-controlled measurements also showed no significant changes.

For $\Phi \gtrsim \Phi_{g}$, the bulk rheology is as previously reported, Fig. 1(a), with flow curves of Herschel-Bulkley (HB) form: $\sigma-\sigma_{y} \propto \dot{\gamma}^{n}$, with $n \simeq 0.4-0.5$. The strong increase of $\sigma_{u}$ with $\Phi$, due to the vanishing of free volume as $\Phi \rightarrow 1$ [32], is consistent with $\sigma_{y}(\Phi) \simeq \sigma_{0}(1-\Phi)^{-p}$ 

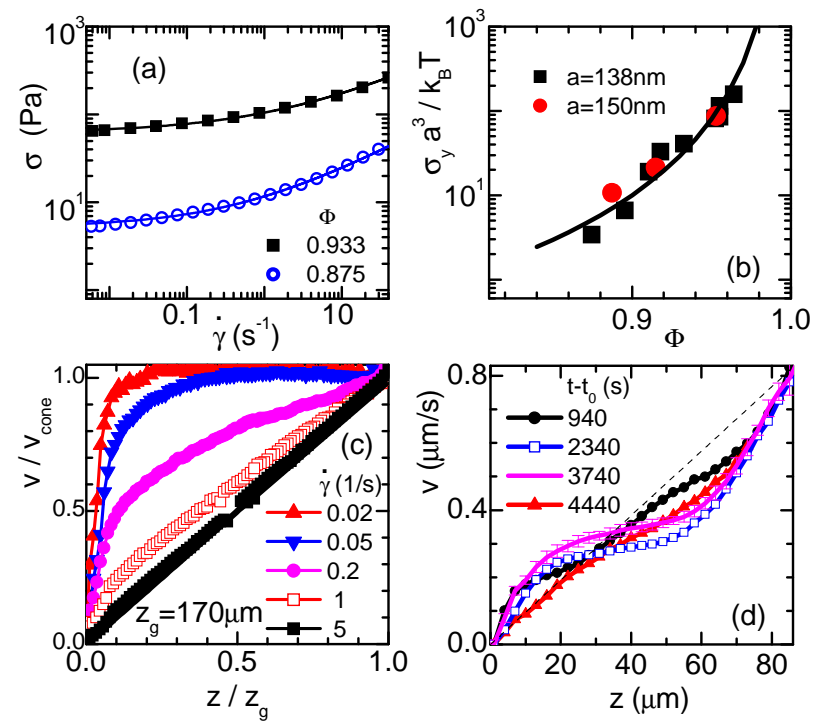

FIG. 1: (a) Flow curves $\sigma(\dot{\gamma})$ with HB fits (lines) for $\Phi=0.875$ and $\Phi=0.933$. (b) Yield stress versus $\Phi$. Line: $\sigma_{y}=\sigma_{0}(1-$ $\Phi)^{-3}$ with $\sigma_{0}=0.01 k_{B} T / a^{3}$. (c) Velocity profiles $v(z)$ for $\Phi=0.933$ at various $\dot{\gamma}$. (d) Evolution of $v(z)$ after startup shear of $\dot{\gamma}=0.01 \mathrm{~s}^{-1}$ at $t_{0}(\Phi=0.94)$; data for $t-t_{0}=3740 \mathrm{~s}$ show error bars. Data in (a),(c) and (d) are for $a=138 \mathrm{~nm}$.

with $p \simeq 3$ and $\sigma_{0} \simeq 0.01 k_{B} T / a^{3}$, Fig. 1(b).

Until now, HB and similar monotonic flow curves for glasses have not been linked to non-transient shear banding. However, the underlying velocity profiles, shown in Fig. 1(c) for $z_{g}=170 \mu \mathrm{m}$ and $\Phi=0.933$, exhibit a marked change when we decrease the imposed shear rate $\dot{\gamma}=\int \dot{\gamma}(z) d z / z_{g}$ (here $\left.\dot{\gamma}(z) \equiv \partial_{z} v\right)$. At large $\dot{\gamma}, v(z)$ is linear, but for $\dot{\gamma}=0.2 \mathrm{~s}^{-1} v(z)$ becomes highly nonlinear, with an enhanced rate near the plate and a progressive reduction towards the cone. For even smaller rates, $\dot{\gamma} \leq 0.05 \mathrm{~s}^{-1}$, the nonlinearity grows and $\dot{\gamma}(z)$ decreases continuously from a value $\gg \dot{\gamma}$ near the plate to $\dot{\gamma}(z) \simeq 0$ for larger $z$. The width of the fluidized band appears to saturate for low $\dot{\gamma}$ at $\sim 80 a$ (not shown); we find no evidence for a minimum strain rate in this band [31]. The behavior for $z_{g}=60 \mu \mathrm{m}$ and $90 \mu \mathrm{m}$ is essentially the same. Such continuously varying flow profiles strongly contrast with the distinct solid and fluid bands in thixotropic yield stress fluids [9, 10]. HS glasses, which show only very weak aging of quiescent properties [32], are thus distinct from such systems. Note from Figs. 1(c) and 2(b) that $v(z)$ has no unique 'symmetry': the fluidized band may appear near either the plate or the cone. This rules out sedimentation or specific wall rheology [16] as explanations 33]. The growth of the fluidized band with $\dot{\gamma}$ also contrasts with the rate dependence (or lack thereof) of the cooperativity length found in [16, 21].

Next, we discuss the concentration dependence of the observed behavior, Fig. 22 For both $\Phi=0.895$ (just within the glass, Fig. 2(a)) and for a much higher concentration $\Phi=0.948$, Fig. 2(b)), we again observe a transition to a nonlinear velocity profile as $\dot{\gamma}$ is lowered, but
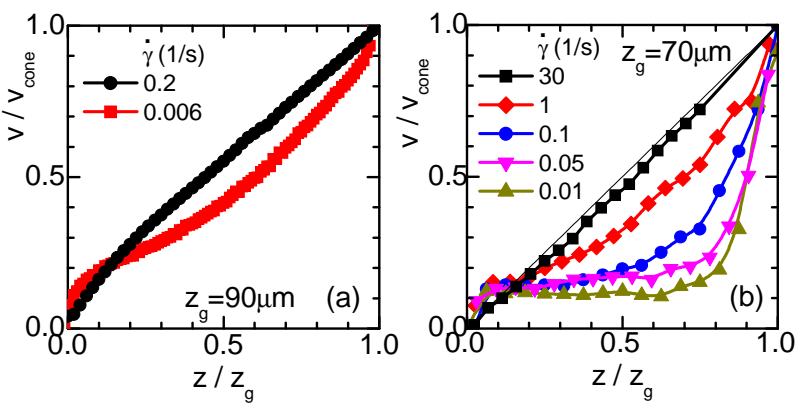

FIG. 2: Velocity profiles at different volume fractions: (a) $\Phi=0.895(a=138 \mathrm{~nm})$, (b) $\Phi=0.948$ ( $a=150 \mathrm{~nm})$.

the shear rate at which this occurs is respectively much smaller and higher than for $\Phi=0.933$, Fig. 1 (c). On decreasing $\dot{\gamma}$, we define the critical shear rate, $\dot{\gamma}_{c}$, to be that point at which the maximum deviation from linearity of the normalized velocity profile, $|v(z)-\dot{\gamma} z| / v_{\text {cone }}$, first exceeds 0.1. Results for the critical Péclet number, $\mathrm{Pe}_{\mathrm{c}}=\dot{\gamma}_{c} \tau_{B}$ (where $\tau_{B}=6 \pi \eta_{s} a^{3} / k_{B} T$ is the Brownian time) are shown as a function of $\Phi$ in Fig. 3 ,

To begin to interpret our observations, we first show that the observed velocity profiles can be reconciled with HB behavior, simply by postulating a small concentration variation $\delta \Phi(z)$ across the gap. Writing the HB form as $\dot{\gamma} \tau=\left[\left(\sigma / \sigma_{y}(\Phi)\right)-1\right]^{1 / n}$ with $\sigma_{y}(\Phi)=\sigma_{0}(1-\Phi)^{-3}$ as before, we can then calculate $v(z, \sigma)=\int_{0}^{z} \dot{\gamma}(\bar{\Phi}+$ $\left.\delta \Phi\left(z^{\prime}\right), \sigma\right) d z^{\prime}$ for a given mean concentration $\bar{\Phi}$ and a choice of $\delta \Phi(z)$. In Fig. 4 (a) we do this for a uniform gradient $\partial \delta \Phi(z) / \partial z=|\delta \Phi| / z_{g}$ with $|\delta \Phi| / \bar{\Phi}=0.002$, at various values of the reduced stress $\sigma / \sigma_{y}(\bar{\Phi})$. When $\sigma$ approaches $\sigma_{y}(\bar{\Phi}), v(z)$ changes from weakly to strongly nonlinear, reflecting the progressive localization of shear within regions of the sample with the lowest yield stress $\sigma_{y}(\Phi(z))$, i.e. with the lowest $\Phi(z)$. These results strikingly resemble the experimental data in Fig. 1(b), although $\delta \Phi$ is too small to be directly measured [34]; different symmetries of $v(z)$ in other experiments can also be explained by corresponding changes in $\delta \Phi(z)$. Note that the mean shear rate $\dot{\gamma}=v\left(z_{g}, \sigma, \Phi(z)\right) / z_{g}$ differs from $\dot{\gamma}(\sigma, \bar{\Phi})$, but the effective flow curves $\sigma(\dot{\gamma}, \Phi(z))$ deviate only slightly from the uniform $\sigma(\dot{\gamma}, \bar{\Phi})$, see Fig. 幽(b).

While concentration gradients can thus account for the results, their origin and the enhanced shear localization with increasing $\bar{\Phi}$ remain to be explained. We now show that both are explicable via the SCC instability scenario of 23]. Fluctuations in concentration $(\delta \Phi)$ and shear rate $(\delta \dot{\gamma})$ evolve via the diffusion and Navier-Stokes equations, in which shear-induced migration and the $\Phi$ dependence of the shear stress must be included [23]. For small fluctuations along $z$ we have (to linear order in $\delta \Phi, \delta \dot{\gamma}$ ):

$$
\begin{aligned}
& \partial_{t} \delta \Phi=-\vec{\nabla} \cdot \vec{J} \simeq M\left(\Pi_{\Phi} \partial_{z}^{2} \delta \Phi+\Pi_{\dot{\gamma}} \partial_{z}^{2} \delta \dot{\gamma}\right), \\
& \partial_{t} \delta \dot{\gamma}=\rho^{-1} \partial_{z}^{2} \sigma \simeq \rho^{-1}\left(\sigma_{\Phi} \partial_{z}^{2} \delta \Phi+\sigma_{\dot{\gamma}} \partial_{z}^{2} \delta \dot{\gamma}\right) .
\end{aligned}
$$

Here we have introduced the shorthand $\Pi_{\dot{\gamma}} \equiv \partial \Pi /\left.\partial \dot{\gamma}\right|_{\Phi}$, likewise $\Pi_{\Phi}, \sigma_{\dot{\gamma}}$ and $\sigma_{\Phi} ; M$ is a collective mobility and 


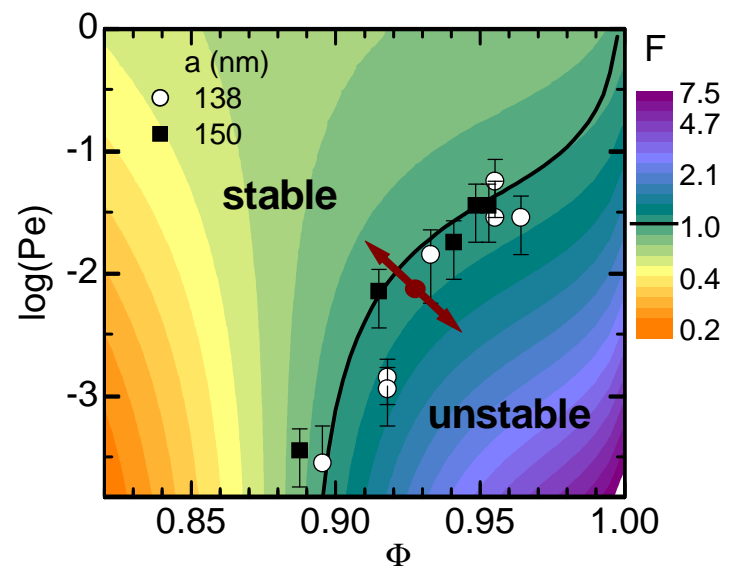

FIG. 3: Line: critical flow rate $\mathrm{Pe}_{\mathrm{c}}(\Phi)$ from Eq. (7) with $[p, r, m=n, A, B]=[3,4,0.4,25,0.005]$. Colors mark the value of $F$. Symbols: experimental values for $\mathrm{Pe}_{\mathrm{c}}$ (averaged over $z_{g}$ ). Arrow: possible evolution of an unstable state.

$\rho$ the density. The migration current $\vec{J}$ arises from particle pressure gradients $\partial_{z} \Pi(\Phi, \dot{\gamma})$ due to variations in both $\Phi$ and $\dot{\gamma}$ 25, 35]. The terms involving $\Pi_{\dot{\gamma}}$ and $\sigma_{\Phi}$ in Eqs. (112) cause respectively particle migration towards regions of low shear rate, and accelerated shear in regions of low concentration; together, these amplify fluctuations. That is, a fluctuation towards higher $\Phi$ in some region creates a lower shear rate there. This promotes inward particle migration, giving a positive feedback effect. This tendency is counteracted by the remaining terms which describe stable diffusive spreading of both particles and momentum (or equivalently shear rate). Rewriting Eqs. (112) as $\partial_{t} \Psi_{i}=L_{i j} \partial_{z}^{2} \Psi_{j}$ with $\Psi_{i}=(\delta \Phi, \delta \dot{\gamma})$, we see that instability sets in when $\operatorname{det} L_{i j}=M\left(\Pi_{\Phi} \sigma_{\dot{\gamma}}-\Pi_{\dot{\gamma}} \sigma_{\Phi}\right) / \rho$ becomes negative, or equivalently, as first derived in [23, 35], when

$$
F \equiv \frac{\Pi_{\dot{\gamma}} \sigma_{\Phi}}{\Pi_{\Phi} \sigma_{\dot{\gamma}}}>1 .
$$

To evaluate $F$, we first write the HB form for $\sigma$ in terms of the Péclet number $\mathrm{Pe}=\dot{\gamma} \tau_{B}$ :

$$
\sigma=\frac{\sigma_{0}}{(1-\Phi)^{p}}\left[1+s(\Phi) \mathrm{Pe}^{n}\right], \quad s(\Phi)=A(1-\Phi)^{n} .
$$

The first term is $\sigma_{y}$ and $n \simeq 0.4-0.5$, as before. Typical values for $A$ from our experiments are $A=10-20$. The particle pressure $\Pi$ for HS colloids has a similar form:

$$
\Pi=\frac{\Pi_{0} \Phi}{(1-\Phi)}\left[1+g(\Phi) \mathrm{Pe}^{m}\right], \quad g(\Phi) \equiv B(1-\Phi)^{1-r} .
$$

Here $\Pi_{0}=2.175 \phi_{m} k_{B} T / \pi a^{3}$; the first term then approximates the osmotic pressure at rest [36], whereas the second term is the contribution due to shear. For the latter, a $\mathrm{Pe}^{2}$ dependence was found in [36, 37], but this is restricted to a linearly viscous regime $(\sigma \propto \mathrm{Pe})$ at very small $\mathrm{Pe}$, in which Eq. 3 yields $F \propto \mathrm{Pe}^{2}$ resulting in stable flow. However, this regime is hard to access, and indeed completely absent whenever $\sigma_{y}$ is nonzero $\left(\Phi>\Phi_{g}\right)$,
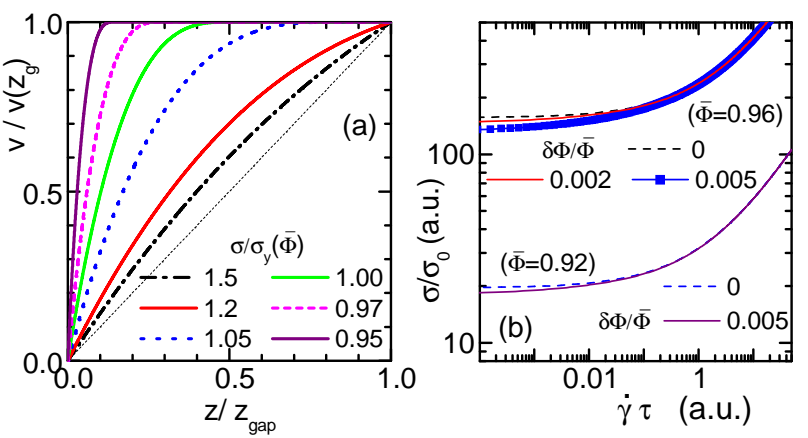

FIG. 4: Velocity profiles $v(z) / v\left(z_{g}\right)$, calculated from $\dot{\gamma} \propto$ $\left[\left(\sigma / \sigma_{y}(\Phi(z))\right)-1\right]^{2}$ and $\sigma_{y} \propto(1-\Phi)^{-3}$, with $\Phi(z)-\bar{\Phi}=$ $|\delta \Phi|\left[\left(z / z_{g}\right)-(1 / 2)\right]$ with $\bar{\Phi}=0.96,|\delta \Phi| / \bar{\Phi}=0.002$, for different $\sigma / \sigma_{y}(\bar{\Phi})$. (b) Effective flow curves $\sigma$ versus $\dot{\gamma}=$ $\left[v\left(z_{g}, \sigma, \Phi(z)\right)\right] / z_{g}$ for various $\bar{\Phi}$ and $|\delta \Phi|$.

where it is replaced by a non-Newtonian regime in which both the stress and the particle pressure increase sublinearly with Pe. From simulations for $\Phi \lesssim \Phi_{g}$ [25, 37], we extract $m=0.4-0.5$, (and $B \simeq 0.003, r \simeq 3$ ), so that $m \simeq n$, as is also observed in simulations of $2 \mathrm{D}$ foams [38]. For $\Phi \gtrsim \Phi_{g}$, and for glassy flow in general, we expect these two exponents to remain similar (e.g., within mode coupling theory, $m=n$ seems probable [20]).

Using Eqs. (45), we obtain a limiting value for $F$ at large $\mathrm{Pe}$ as $F_{\infty}=m(p-n) / n r$ [39]. The flow is thus stable in this regime provided $r>p-n$; to explain our experiments we require $r \gtrsim 2.5$. More interesting is the result for small $\mathrm{Pe}$, where we obtain

$$
F \rightarrow \mathrm{Pe}^{m-n} \frac{m p g(\Phi) \Phi}{n s(\Phi)} \simeq \frac{m p B \Phi}{n A(1-\Phi)^{r+n-1}}=F_{0}
$$

Here $F_{0}(\Phi)$ is a quasi-plateau value maintained while $\mathrm{Pe}^{m-n} \simeq 1$ (and a true limiting value if $m=n$ ). It follows that homogeneous flow at low Pe is unstable for concentrations $\Phi>\Phi_{c}$, where $F_{0}\left(\Phi_{c}\right)=1$. We argue that it is this SCC-induced instability that creates the small variations $\delta \Phi(z)$ that were assumed in Fig. 4(a), and which account for the experimentally observed localized flow at small $\dot{\gamma}$. Because, as in [23], our analysis is limited to linear stability, we do not have a clear idea of the mechanism limiting the growth of $\delta \Phi$. However, the extreme nonlinear dependence of both $\sigma$ and $\Pi$ on $\Phi$ as $\Phi \rightarrow 1$ makes it plausible that $\delta \Phi$ remains small.

The critical flow rate $\mathrm{Pe}_{\mathrm{c}}$, below which the instability sets in, follows from the condition $F\left(\mathrm{Pe}_{\mathrm{c}}, \Phi\right)=1$. For the case $m=n$ this simplifies to [39]:

$$
\operatorname{Pe}_{\mathrm{c}}(\Phi) \simeq\left[\frac{p}{r s(\Phi)\left(1-F_{\infty}\right)}\left(1-\frac{1}{F_{0}(\Phi)}\right)\right]^{1 / n}
$$

Figure 3 shows $\operatorname{Pe}_{c}(\Phi)$ for specific parameter values. Entering the unstable regime $\Phi>\Phi_{c}, \mathrm{Pe}_{c}$ increases and grows $\sim s(\Phi)^{-1 / n}$ for $\Phi \rightarrow 1$. Our model is able to give a quantitative account of the data; the fit [40] gives 
$\Phi_{c}=0.89$, close to, but above, $\Phi_{g}$. (Note that Eq. (6) does not rule out $\Phi_{c}<\Phi_{g}$ in principle.)

In contrast to most shear banding scenarios [1], the present system seems unable to achieve global stability by separating into distinct bands. This is illustrated by the arrows in Fig. 3. an initially unstable state can form a locally depleted region, $\Phi(z)<\bar{\Phi}$, that is stabilized when $\operatorname{Pe}(\Phi(z))>\mathrm{Pe}_{c}$, but the remaining concentrated region is even more unstable than before. This suggests that the banded flow should have residual temporal fluctuations. We have indeed observed this (Fig. 1(d)); after startup shear of $\dot{\gamma}=0.01 \mathrm{~s}^{-1}$, a weakly nonlinear profile develops a central region where $\dot{\gamma}(z)$ is strongly reduced, which then speeds up by expanding the lower band, and subsequently reverts to a larger $\dot{\gamma}(z)$. We have also observed (in a planar shear cell [31]) that a fluidized band can swap from $z \simeq 0$ to $z \simeq z_{g}$ over sufficient time. Leaving a detailed study of these effects for future work, we note that the shear banding we observe is permanent (if unsteady) not transient; moreover, our stress is timeindependent, in contrast to the results in [17].

Our shear-banding mechanism arises from the concentration dependent nonlinear rheology of glasses along with the nonlinear process of 'Brownian dilation' whereby flow increases the particle pressure $\Pi(\Phi$, Pe $)$. For larger
Pe than those studied here, hydrodynamic effects lead to a much stronger, linear increase of $\Pi$ with flow rate [26], ultimately leading to shear thickening in these suspensions. Hence, the localization we observe might be interpretable as a precursor to shear thickening.

In conclusion, we have shown that HS colloidal glasses exhibit a new type of shear banding, well described by a model in which SCC leads to unstable flow near yielding. In this scenario, very small concentration variations can sustain large variations in flow rate. Our results may also be relevant for flow in other glassy materials, such as foams where 'dilatancy' has recently been observed [41]. It may also shed light on shear bands and dilatancy in metallic glasses [5]. We mention in particular simulations of a model glass [18], which showed both fluctuating shear bands and small but finite concentration gradients.

We thank A.B. Schofield for colloids, and J. Brader, P. Coussot, D. Marenduzzo, A. Morozov, H. Tanaka, M. van Hecke and T. Voigtmann for discussions. Work funded in part by EPSRC EP/D067650 and EP/E030173. L.I. was funded by EU MRTN-CT-2003-504712. G.P. and P.B. acknowledge EU funding from ToK 'Cosines' (MTCDCT-2005-029944) and NMP Small 'Nanodirect' (CPFP7-213948-2). M.E.C. is funded by the Royal Society.
[1] M. E. Cates and S. M. Fielding, Adv. Phys. 55, 799 (2006); P. D. Olmsted, Rheol. Acta, 47, 283 (2008); J. K. G. Dhont and W. J. Briels, Rheol. Acta 47, 283 (2008).

[2] G. Ovarlez et al., Rheol. Acta 48, 831 (2009).

[3] H. M. Jaeger et al. , Rev. Mod. Phys. 68, 1259 (1996); D. Fenistein and M. van Hecke, Nature 425, 256 (2003).

[4] J. W. Rudnicki, J. Mech. Phys. Solids, 23, 371 (1975).

[5] F. Spaepen, Act. Metall. 25, 407 (1977); ibid 30, 447 (1982); J. Li et al., Phil. Mag. A, 822623 (2002).

[6] S.M. Fielding and P.D. Olmsted, Eur. Phys. J. E, 11, 65 (2003); M.E. Helgeson et al., Phys. Rev. Lett. 105, 084501 (2010).

[7] P. Coussot et al., Phys. Rev. Lett. 88218301 (2002).

[8] S. A. Rogers et al., Phys. Rev. Lett. 100, 128304, (2008).

[9] P. Moller et al., Phys. Rev. E 77, 041507 (2008).

[10] V. Bertola et al., J. Rheol. 47, 1211 (2003).

[11] G. Picard et al., Phys. Rev. E 66, 051501 (2002); S. M. Fielding et al., Soft Matter 5, 2378 (2009).

[12] P. Coussot et al., Phys. Rev. Lett. 88175501 (2002).

[13] T. G. Mason et al., J. Col. Int Sci. 179, 439 (1996); F. Rouyer et al., Phys. Rev E 67, 021405 (2003); L. Becu et al., Phys. Rev. Lett 96, 138302 (2006), see also [15].

[14] G. Katgert et al., Europhys. Lett. 90, 54002 (2010).

[15] G. Ovarlez et al., Phys. Rev. E 78036307 (2008).

[16] J. Goyon et al., Nature 454, 84 (2008).

[17] T. Divoux et al., Phys. Rev. Lett. 104208301 (2010).

[18] F. Varnik et al., Phys. Rev. Lett. 90, 095702 (2003).

[19] Y. Shi et al., Phys. Rev. Lett. 98, 185505 (2007).

[20] J. M. Brader et al., Proc. Nat. Acad. Sci. USA, 106, 15186 (2009); Phys. Rev. Lett. 101, 138301 (2008); M. Fuchs, M. Cates, Phys. Rev. Lett. 89, 248304 (2002).

[21] L. Bocquet et al., Phys. Rev. Lett. 103, 036001 (2009).
[22] R. Yamamoto, A. Onuki, Phys. Rev. E 58, 3515 (1998); G. Picard et al., Phys. Rev. E 71, 010501 (2005); A. Lemaître, C. Caroli, Phys. Rev. Lett. 103, 065501 (2009).

[23] V. Schmitt et al., Phys. Rev. E 52, 4009 (1995).

[24] P.R. Nott, J.F. Brady, J. Fluid Mech. 275, 157 (1994).

[25] Y. Yurkovetsky and J.F. Morris, J. Rheol. 52, 141 (2008).

[26] A. Deboeuf et al., Phys. Rev. Lett. 102, 108301 (2009).

[27] A. Furukawa and H. Tanaka, Nature 443, 434 (2006); Nature Mat. 8, 601 (2009).

[28] W. van Megen et al., Phys. Rev. E 58, 6073 (1998).

[29] P. Ballesta et al., Phys. Rev. Lett. 101, 258301 (2008).

[30] R. Besseling et al., Adv. Coll. Int. Sci. 146, 1 (2009).

[31] R. Besseling et al., Phys. Rev. Lett. 99, 028301 (2007).

[32] N. Koumakis et al., Soft Matter 4, 2008; K.S. Schweizer, G. Yatsenko, J. Chem. Phys. 127, 164505 (2007).

[33] In our geometry $\sigma(z)$ is uniform within $0.03 \%$.

[34] The typical $|\delta \Phi| / \bar{\Phi}$ needed to mimic $v(z)$ in experiments corresponds to $\delta \phi \lesssim 0.3 \%$, below our confocal imaging resolution ( $\gtrsim 0.5 \%)$. MRI experiments as in [15] may be able to detect this. The associated change in the measured $\sigma_{y}$ is $\delta \sigma_{y} / \sigma_{y}(\bar{\Phi}) \simeq(p|\delta \Phi| / 2) /(1-\bar{\Phi}) \lesssim 10 \%$. Due to the related experimental uncertainty in $\sigma_{y}(\bar{\Phi})$, it is dificult to extract $\delta \Phi(z)$ from the measured $v(z)$ near $\sigma_{y}$.

[35] In [23], $J$ was described via a chemical potential $\mu(\Phi, \dot{\gamma})$. Here we use $J=-M\left[\partial_{\Phi} \Pi \nabla \Phi+\partial_{\dot{\gamma}} \Pi \nabla \dot{\gamma}\right]$ as in [25, 26].

[36] J.F. Brady and M. Vicic, J. Rheol. 39, 545 (1995).

[37] R. Foss and J.F. Brady, J. Rheol. 44, 629 (2000).

[38] V.J. Langlois et al., Phys. Rev. E 78, 021401 (2008).

[39] We have used the fact that $1-\Phi \ll r \Phi$.

[40] In the fit we fix $p=3, m=n$, and vary $A, B, n$ and $r$.

[41] S.P.L. Marze et al., Colloids Surf. A 263, 121 (2005). 